\title{
Multiphysics modeling of magnetorheological dampers
}

\author{
David Case, Behzad Taheri and Edmond Richer* \\ Biomedical Instrumentation and Robotics Laboratory, \\ Southern Methodist University
}

\begin{abstract}
The dynamics of a small scale magnetorheological damper were modeled and analyzed using multiphysics commercial finite element software to couple the electromagnetic field distribution with the non-Newtonian fluid flow. The magnetic flux lines and field intensity generated within the damper and cyclic fluid flow in the damper under harmonic motion were simulated with the AC/DC and CFD physics modules of COMSOL Multiphysics, respectively. Coupling of the physics is achieved through a modified Bingham plastic definition, relating the fluid's dynamic viscosity to the intensity of the induced magnetic field. Good agreement is confirmed between simulation results and experimentally observed resistance forces in the damper. This study was conducted to determine the feasibility of utilizing magnetorheological dampers in a medical orthosis for pathological tremor attenuation. The implemented models are thus dimensioned on a relatively small scale. The method used, however, is not specific to the damper's size or geometry and can be extended to larger-scale devices with little or no complication.
\end{abstract}

\section{INTRODUCTION}

Variable damping through the use of magnetorheological (MR) fluids is a strategy currently being used commercially in vehicle shock absorbers and seismic vibration dampers for civic structures [1], [2]. In the field of human-machine interaction, particularly that of wearable robotics, high strength-to-weight ratio actuators are required to maximize assistive and rehabilitative potential [3]. MR-based actuators can potentially achieve these high ratios and have the additional advantages of rapid response time and high fidelity control [4], [5]. In orthotic applications, these characteristics allow MR fluid dampers to be tuned to the individual needs of a patient and adjusted if the patient's condition changes. Moreover their rapid dynamic response makes them suitable for use in active devices that vary the damping in real-time. This study was conducted for the optimization of a custom MR damper for use in an active medical orthosis for the attenuation of pathological tremor [6]. Thus the models developed are on a relatively small scale (dimensions are presented in the following section). The method used, however, could potentially be extended to larger-scale devices without difficulty.

A magnetorheological fluid (MRF) consists of a suspension of microscopic magnetizable particles in a non-magnetic carrier medium, usually water or some type of synthetic oil. When there is no magnetic field applied to the fluid space, the fluid behaves in a roughly Newtonian manner. Applying a magnetic field causes the microscopic particles suspended in the fluid to become uniformly oriented and form chains along the magnetic flux lines. This

${ }^{\star}$ Corresponding author E-mail: richer@lyle.smu.edu 
temporary internal structure changes the fluid's rheological behavior. When flow occurs perpendicularly to the magnetic flux lines, the resistance of the microparticle chains causes the fluid to exhibit a nonuniform yield stress. Thus, when a magnetic field is applied to a MRF within a narrow channel, the flux lines spanning the gap, the fluid behaves similarly to a Bingham plastic. Since the observed yield stress is directly related to the intensity of the magnetic field, MRFs are ideally suited for use in low-power tunable dampers.

Design and evaluation of such dampers prior to their construction requires a high fidelity model with wide scope. This modeling is problematic, however, due partially to the inherent nonlinearities of fluid dampers in general and partially to the piecewise nature of the mathematical description of Bingham plastics. As a result, the majority of dynamic models to date have been phenomenological in nature [7], [8]. In the following sections of this paper the authors explore a multiphysics finite element approach to the static and dynamic modeling problems described and provide data from experiments on a prototype damper for model validation.

\section{DIMENSIONS AND APPLICATIONS}

The MR dampers examined in this project were designed for application to a medical orthosis for the mechanical suppression of pathological tremor in the human wrist. Dampers of the general design proposed here would be mounted above the dorsal and radial surfaces of the forearm, with the moving rods connected to the hand using articulated linkages and an orthotic glove. Considering the biomechanical characteristics of tremorous movement, the required resistance force produced by the damper would be approximately $37 \mathrm{~N}$, and the velocity of the rod is projected in the range of $0.5-2.0 \mathrm{~m} / \mathrm{s}[6]$.

A piston/cylinder configuration with one axial coil was adopted for the dampers due to its ease of manufacturing. The magnetic field produced by the copper coil wound about the middle section of the piston spans the annular gap between the piston and cylinder (Figure 1). Both the cylinder wall and the piston head were machined from magnetically permeable material to close the magnetic circuit and direct the magnetic flux lines across the piston/cylinder gap.

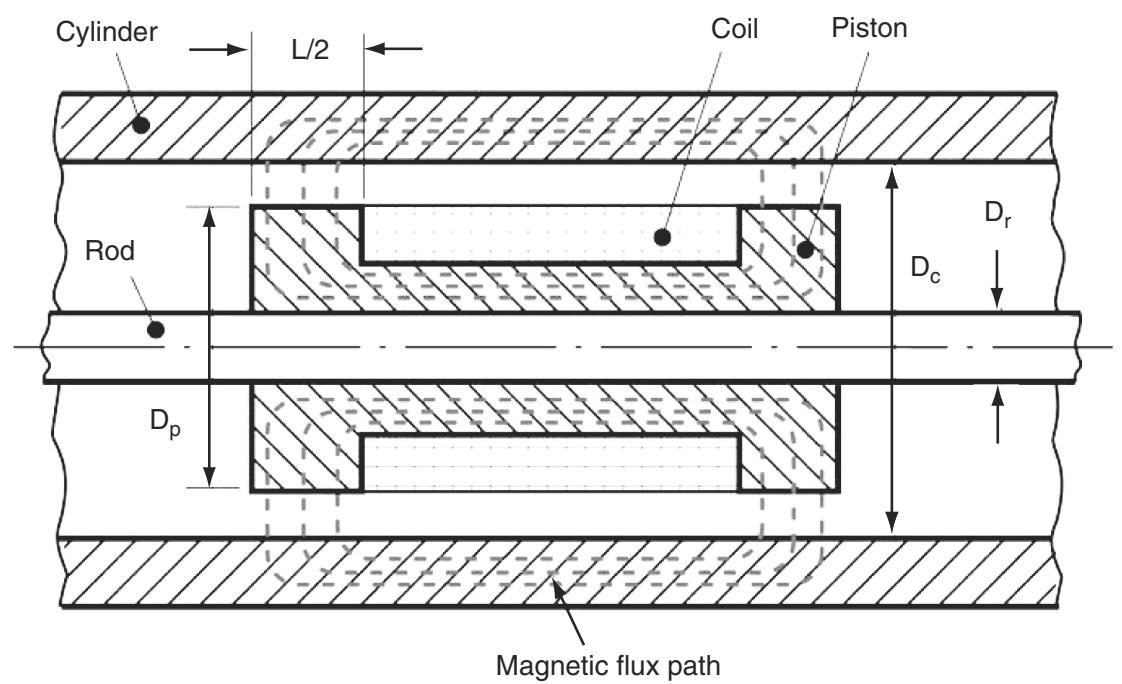

Figure 1 Cross section diagram of a piston/cylinder magnetorheological damper design. 
Table 1 MR Damper geometric and Functional Parameters.

\begin{tabular}{lcc} 
Parameter & Symbol & Value \\
\hline Cylinder Bore Diameter & $\mathrm{D}_{\mathrm{c}}$ & $12.7 \mathrm{~mm}$ \\
\hline Rod Diameter & $\mathrm{D}_{\mathrm{r}}$ & $4.76 \mathrm{~mm}$ \\
\hline Piston Diameter & $\mathrm{D}_{\mathrm{p}}$ & $11.43 \mathrm{~mm}$ \\
\hline Active Piston Length & $\mathrm{L}_{\mathrm{m}}$ & $7.94 \mathrm{~mm}$ \\
\hline Total Piston Length & $\mathrm{L}_{\mathrm{t}}$ & $16.67 \mathrm{~mm}$ \\
\hline Piston/Cylinder Gap & $\mathrm{h}$ & $0.64 \mathrm{~mm}$ \\
\hline Stroke Length & $\mathrm{L}_{\mathrm{s}}$ & $60.0 \mathrm{~mm}$ \\
\hline Max Coil Current & $\mathrm{i}_{\max }$ & $700 \mathrm{~mA}$ \\
\hline Max Power Consumption & $\mathrm{P}_{\max }$ & $6 \mathrm{~W}$ \\
\hline Number of Coil Turns & $\mathrm{N}_{\text {coil }}$ & 420 \\
\hline Wire Cross-Section & $\mathrm{A}_{\text {coil }}$ & $0.0324 \mathrm{~mm}$ \\
\hline Coil Inductance & $\mathrm{L}_{\text {coil }}$ & $5.1 \mathrm{mH}$ \\
\hline
\end{tabular}

The primary geometric parameters that determine the resistance force in this design are the piston area, gap cross-section and its active length. The values used for the custom design examined in this study are presented in Table I. The variable parameters are the fluid's viscosity and yield strength, the latter being dependent upon the generated magnetic field and thus upon the dimensions of the coil and the applied current. These variable parameters are discussed in more detail in the following sections.

\section{METHODS AND ASSUMPTIONS}

Viscosity regularization for the modeling of Bingham plastic flow is not a new concept. A few examples of past work include that of [9], [10], and [11], though a more comprehensive list is presented by [12]. Two major issues arise with the use of the regularization method. First, of course, is the potential for a break down at the computational level. Since the classical Bingham plastic definition is piecewise defined, singularities may arise when attempting to solve a fluid flow model with strong adherence to this definition. And second is lack of fidelity of the model when the approximation is weak.

This first concern may be circumvented by sufficient refining of the model mesh. While taxing on computer resources, this approach becomes continually more feasible as hardware development progresses. The second concern has been addressed notably by [12] and [13]. The results of error analysis in each of these studies suggest that regularization methods tend to produce spurious results in hydrodynamic and static stability problems. However, it is shown that in situations where there are no stagnant fluid regions (e.g. the shear stress within the fluid is consistently in excess of the yield point) regularized models can produce fairly accurate calculations of the flow profile.

For this study, no friction due to sealing was assumed in the FEM simulation. For validation purposes, the friction component was removed from the experimental data as described in Section 5.

\section{A. SIMULATION PHYSICS}

Finite element simulations were performed with COMSOL Multiphysics (COMSOL, Inc., Los Angeles, CA), using the Electromagnetics and Fluid Flow physics modules. As a basis for a time-dependent solver, the relations defined by Maxwell's equations were employed, including the equation of continuity for constant electric charge density, 
$\nabla \cdot J=0$

and Ampere's law,

$$
\nabla \times H=J
$$

Here, $H$ is the magnetic field intensity and $J$ the current density. Constitutive relations are established between magnetic field intensity and magnetic flux density, $B$,

$$
B=\mu_{o} \mu_{r} H
$$

and between current density and electric field intensity, $E$,

$$
J=\sigma E+J_{e}
$$

In these equations, $\mu_{r}$ and $\mu_{0}$ are the material permeability and the permeability of free space, respectively, and $\sigma$ is electrical conductivity. The two potentials are described as direct consequences of Gauss' law,

$$
E=-\nabla V
$$

and Faraday's law,

$$
B=\nabla \times A
$$

where $V$ is the electric scalar potential, and $A$ is the magnetic vector potential.

Finally, the $J e$ term from Eqn 4 was generated with COMSOL's Multi-Turn Coil Domain interface, the value representing the external contribution of the electromagnetic coil to the ambient current density,

$$
J_{e}=\frac{N_{\text {coil }} I_{\text {coil }}}{A} \Phi
$$

Here, $N$ is the number of turns in the electromagnetic coil, and $I_{c o i l}$ is the applied current.

For the purpose of simulating the behavior of a magnetorheological fluid, a direct relationship was defined between the fluid's dynamic viscosity and the intensity of the local magnetic field. This viscosity regularization and the general fluid flow dynamics of the model are discussed in detail in the following section.

\section{B. VISCOSITY REGULARIZATION}

Previous work modeled the properties of the MR fluid using the classical Bingham plastic definition and systems of equations described by [14] and [15]. The non-Newtonian fluid properties were assumed to be homogeneous, the flow fully-developed, and fluid inertia negligible [6]. The model was therefore deemed suitable to predict the operational range of the damper but inadequate to accurately predict behavior in oscillatory flow, which would be seen in applications such as real-time tremor attenuation. 
In order to develop an effective control strategy for the suppression of tremor with MR dampers, an accurate dynamic model is needed. However, solving a multi-dimensional Bingham plastic flow problem presents challenges due to the piecewise mathematical description of the fluid [16]:

$$
\begin{aligned}
& \sigma_{i j}=\left(\frac{\sigma_{y}}{\dot{\gamma}}+\eta_{0}\right) \dot{\epsilon}_{i j} \quad \sigma>\sigma_{y} \\
& \dot{\epsilon}_{i j}=0 \quad \sigma>\sigma_{y}
\end{aligned}
$$

Here, $\sigma_{i j}$ represents the deviatoric stress tensor, $\sigma_{y}$ is the yield stress of the fluid, and $\eta_{0}$ is its base viscosity. The term $\dot{\epsilon}_{i j}$ is the rate of strain tensor, and $\dot{\gamma}$ is the strain rate magnitude, given by

$$
\dot{\gamma}=\sqrt{\frac{1}{2} \dot{\epsilon}_{i j} \cdot \dot{\epsilon}_{i j}}
$$

This description of the stress tensor leads to complications in computation, due to its nondifferentiability in the unyielded regions of the fluid. Also, in the case of modeling MR fluids, there is the added complication of a nonuniform yield stress. This variable must be dependent upon the magnetic field intensity and is thus indirectly dependent upon coordinate location.

One approach to the non-differentiability problem is to first determine the bounds of the unyielded regions then to treat them as "plug flow" (a region in which the velocity is a constant vector). This approach, however, assumes fully developed flow and becomes far more complicated when dealing with complex channel geometries [13].

The approach used in this study follows a close approximation of Bingham plastic behavior, similar to that used by [9] and [17]. In these previous works, the unyielded regions were replaced by minimally yielded regions by substituting a continuously differentiable tensor definition for Eqns (8) and (9), such as

$$
\sigma_{i j}=\left(\frac{\sigma_{y}}{\sqrt{\alpha^{2}+\dot{\gamma}^{2}}}+\eta_{0}\right) \dot{\epsilon}_{i j}
$$

The parameter $\alpha$ is a small constant which eliminates the discontinuity that would otherwise appear when differentiating $\sigma_{i j}$. This modified Bingham definition approaches the classical definition as $\alpha \rightarrow 0$.

However, for a time-dependent solver the steep slope at the origin can be problematic without excessive refinement of the time step and mesh. Therefore a further modification of the tensor definition that employs a sigmoid function to mitigate the slope and prevent potential singularities from arising is proposed. With this modification the definition becomes 


$$
\sigma_{i j}=\left(\frac{\sigma_{y} \tanh (\zeta \dot{\gamma})}{\sqrt{\alpha^{2}+\dot{\gamma}^{2}}}+\eta_{0}\right) \dot{\epsilon}_{i j}
$$

Where $\zeta$ is a scaling term for the slope, the definition approaching Eqn (11) as $\zeta \rightarrow \infty$, as seen in Figure 2.

It can be argued that this formulation allows creep of the fluid at stresses below the stated yield point. However, since the MR damper has a relatively simple geometry, and the pressure gradient is expected to change rapidly, there are no projected stagnant regions, and thus this effect can be assumed negligible.

One may note that, with this continuously differentiable description, the fluid behavior may be modeled in the typical manner for non-Newtonian fluids with variable viscosity. Thus, in this model the Navier-Stokes equations are used to describe the fluid motion, assuming fluid incompressibility and a viscosity subject to

$$
\eta=\frac{\sigma_{y} \tanh (\zeta \dot{\gamma})}{\sqrt{\alpha^{2}+\dot{\gamma}^{2}}}+\eta_{0}
$$

The equations of motion can be derived directly from the Cauchy momentum equation, assuming incompressibility,

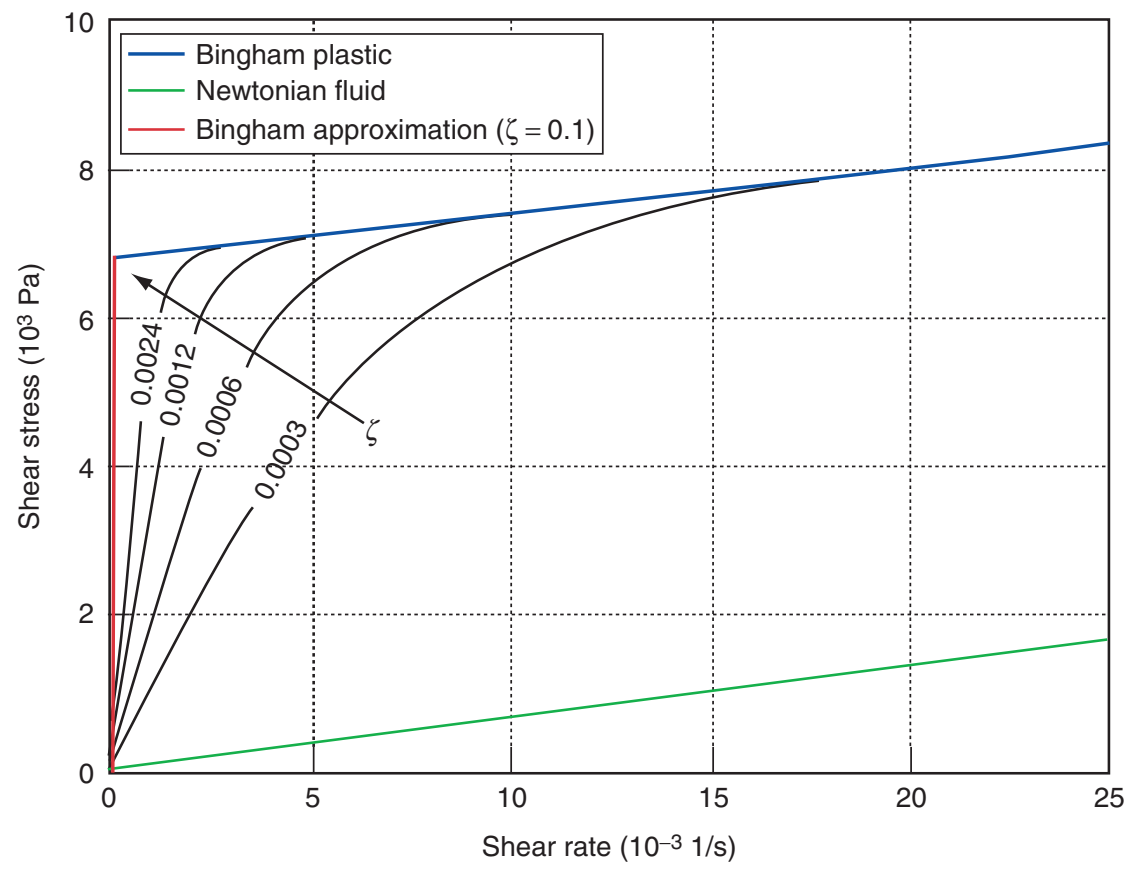

Figure 2 Stress versus strain rate in unidirectional flow for Newtonian fluids and the standard and modified Bingham definitions. 


$$
\begin{aligned}
& \rho=\frac{D u}{D t}=\nabla T+\rho g \\
& \nabla u=0
\end{aligned}
$$

Here, $T$ represents the stress tensor, $u$ represents the velocity vector, and $\rho g$ is the body force due to gravity. In cylindrical coordinates for an axially symmetric model this is

$$
\begin{gathered}
\rho \frac{D u_{r}}{D t}=-\frac{\partial P}{\partial r}+\frac{1}{r} \frac{\partial\left(r \sigma_{r r}\right)}{\partial r}+\frac{\partial \sigma_{z r}}{\partial z}-\frac{\sigma_{\theta \theta}}{r} \\
\rho \frac{D u_{z}}{D t}=-\frac{\partial P}{\partial z}+\frac{\partial\left(r \sigma_{z z}\right)}{\partial z}+\frac{1}{r} \frac{\partial[(\sigma)]_{r z}}{\partial r}
\end{gathered}
$$

where the strain rate elements of Eqn 12 are

$$
\begin{aligned}
& \dot{\epsilon}_{r r}=\frac{\partial u_{r}}{\partial r} ; \dot{\epsilon}_{z z}=\frac{\partial u_{z}}{\partial z} ; \dot{\epsilon}_{\theta \theta}=\frac{u_{r}}{r} ; \\
& \dot{\epsilon}_{r z}=\dot{\epsilon}_{z r}=\frac{1}{2}\left(\frac{\partial u_{r}}{\partial z}+\frac{\partial u_{z}}{\partial r}\right) ; \\
& \dot{\epsilon}_{z \theta}=\dot{\epsilon}_{\theta z}=\dot{\epsilon}_{r \theta}=\dot{\epsilon}_{\theta r}=0
\end{aligned}
$$

\section{MATERIAL PROPERTIES AND MODEL DEFINITION}

Material properties for the MR fluid were chosen based on typical values reported in the manufacturer's provided literature. Viscosity in this case is specified with no ambient magnetic field. Likewise, the nonlinear relationships between yield stress and magnetic field strength as well as magnetic field strength and flux intensity (HB curve) of the MR fluid were initially adopted from the manufacturer's literature and illustrated in Figure 3. The constant electromagnetic and other material properties selected for simulation are presented in Table II. Based on these characteristics of the MR fluid, a value dependent upon the local magnitude of the magnetic field was assigned to the yield stress from Eqn (13), $\sigma_{y}$, at any given point in the fluid region.

The dynamic model was implemented using COMSOL's 2D axisymmetric space with the dimensions presented previously. A deforming mesh was used to simulate the axial translation of the piston. Fluid flow was modeled with the Navier-Stokes equations, assuming incompressibility and the viscosity variation described in the previous section. The Dirichlet boundary conditions assume no slip and no penetration at the rigid boundaries.

The Multi-Turn Coil Domain utilized in the simulation was modeled on the actual dimensions of the copper coil of the damper. The coil has 420 turns in 10 layers, using an AWG 32 gauge copper wire with a conductor cross-sectional area of $3.24 \times 10^{-8} \mathrm{~m}^{2}$. 
(a)

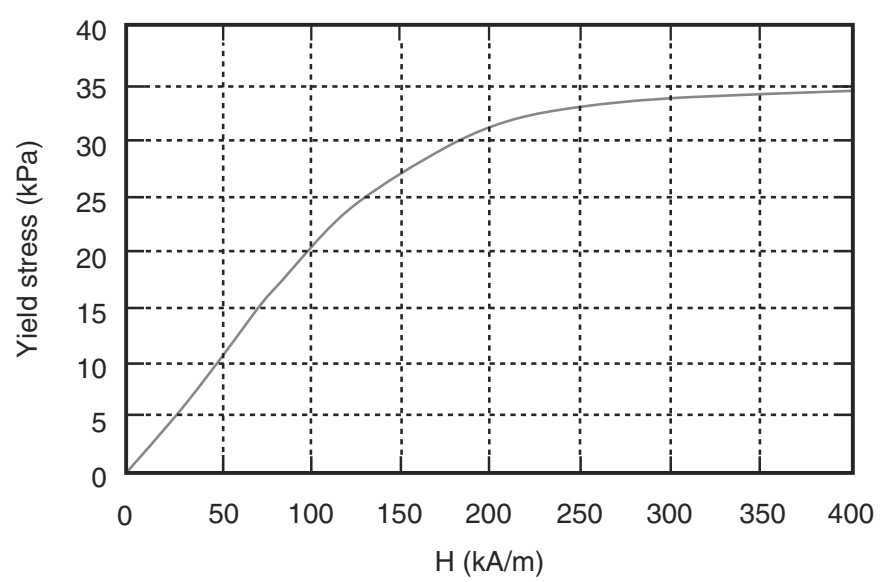

(b)

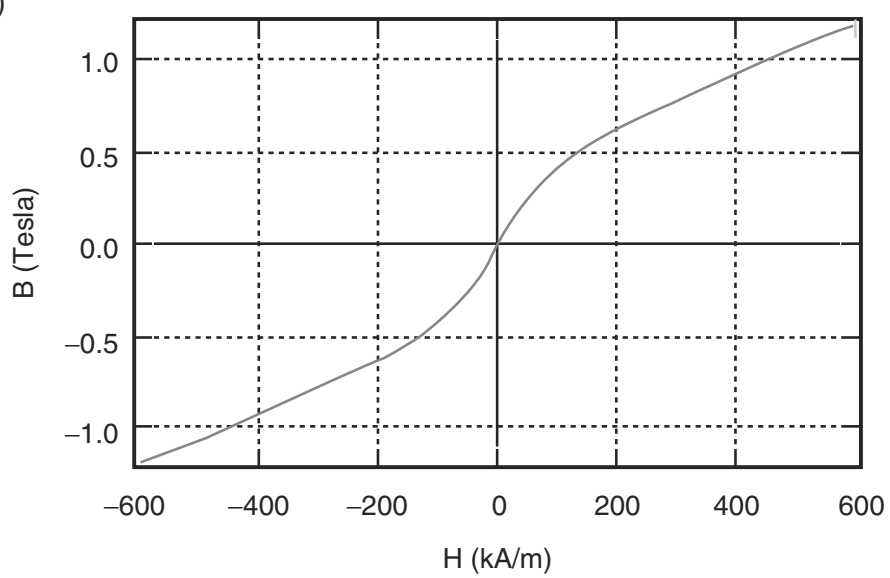

Figure 3 Typical magnetic properties of the MR fluid, MRF-122EG. (a) Yield stress vs. magnetic field strength; (b) magnetic field strength vs. flux intensity.

Table 2 Material Property Constants.

Parameter

Value

\begin{tabular}{lrl}
\hline Piston/Cylinder Relative Permeability & 100 & \\
\hline Rod Relative Permeability & 1 & \\
\hline Coil Relative Permeability & 1 & \\
\hline Fluid Density & 2,380 & $\mathrm{~kg} / \mathrm{m}^{3}$ \\
\hline Fluid Base Viscosity & 0.040 & $\mathrm{~Pa} \cdot \mathrm{s}$ \\
\hline Fluid Conductivity & $3.3 \times 10^{-8}$ & $\mathrm{~S} / \mathrm{m}$ \\
\hline
\end{tabular}

A close-up of the model geometry is shown in Figure 4, the subdomains being the piston $\operatorname{rod}(A)$, the piston head (B), the magnetic coil (C), the MR fluid (D), and the cylinder wall (E). The z-axis is the axis of radial symmetry.

The small-scale damper that is the subject of this paper was designed to function with a maximum coil current of $700 \mathrm{~mA}$, which corresponds to a magnetic field of approximately 
(a)

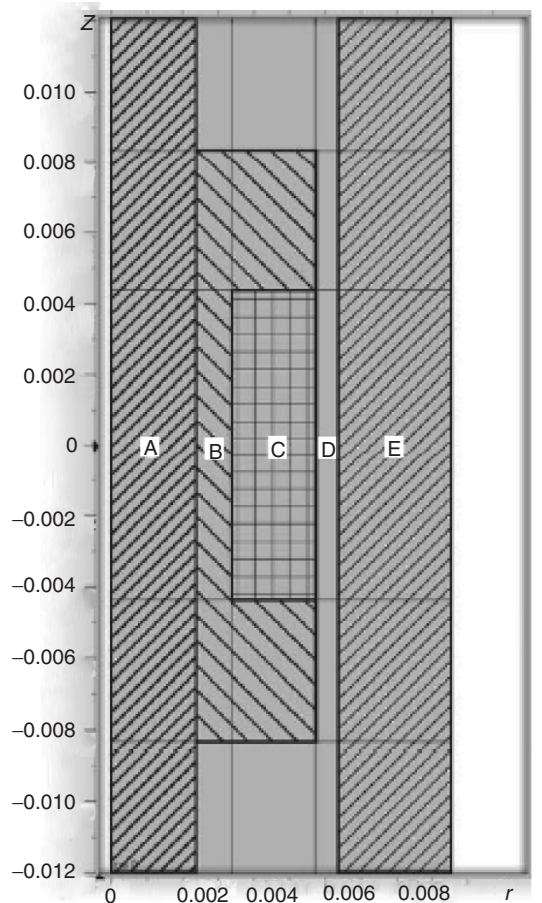

(b)

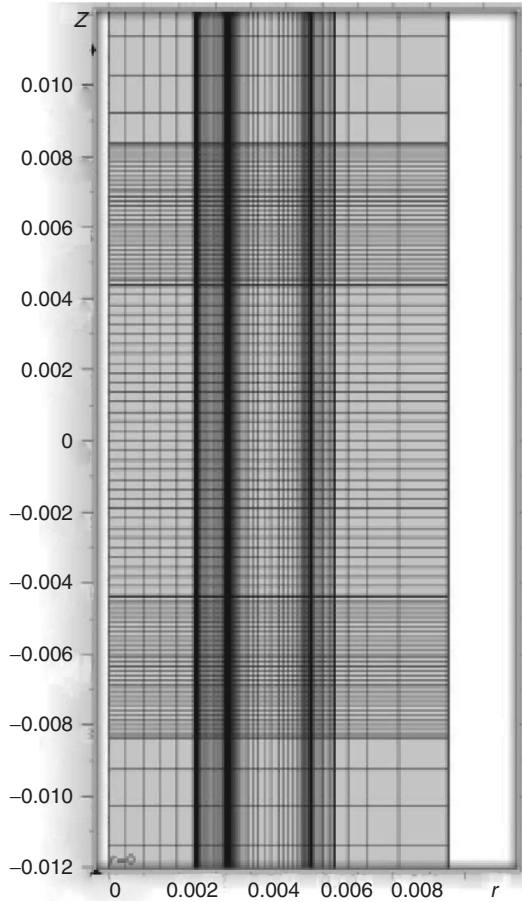

Figure 4 Model domains (a) and mesh (b). The z-axis is the axis of radial symmetry.

$100 \mathrm{kA} / \mathrm{m}$ in the piston/cylinder gap. The yield stress vs magnetic field strength relationship of the MR fluid proved to be highly nonlinear within this region of low field intensity. The actual relationship within this range, shown in Figure 5, has been interpolated from static experiments by applying constant currents within the operational range of the coil and slowly increasing the force applied to the piston rod until yield was observed [6].

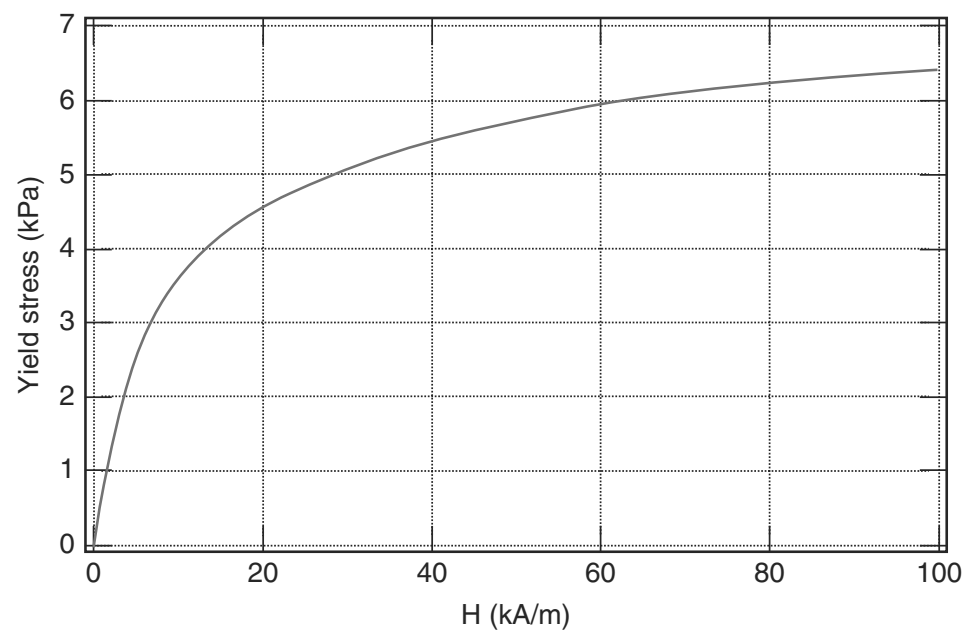

Figure 5 Interpolated yield stress for the MR fluid for weak magnetic field intenstiy. 
In the simulations, the translation of the piston head was defined as a sine function with frequency ranging from $0.5-6.0 \mathrm{~Hz}$ and $25 \mathrm{~mm}$ amplitude. The dynamic viscosity constants of Eqn (12) were specified as $\alpha=0.03$ and $\zeta=0.1$. A parameter sweep was run with coil current ranging from $0-700 \mathrm{~mA}$ with $100 \mathrm{~mA}$ steps. A sample of the simulation results with and without current applied to the magnetic coil is presented in Figure 6. The application of an intense magnetic field to the active portion of the piston/cylinder gap significantly changes the surface shear (strain) rate of the fluid. Consequently, there is a change in dynamic viscosity and thus a more uniform velocity profile observed in the annular gap between piston and cylinder, consistent with what [14] describes as "plug flow" for Bingham plastics.

(a)

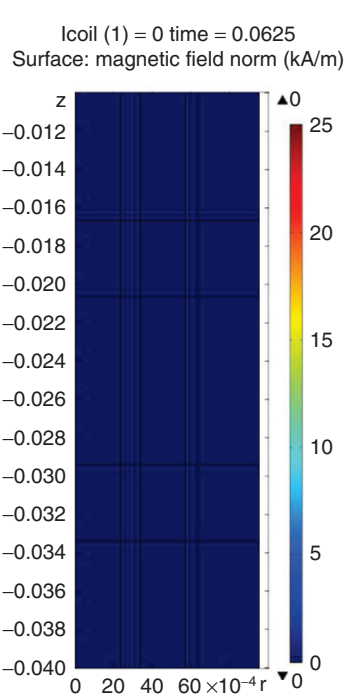

(d)
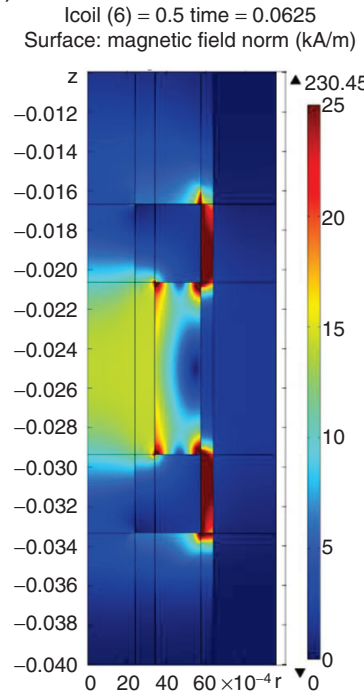

(b) Icoil $(1)=0$ time $=0.0625$
Surface: velocity magnitude $(\mathrm{m} / \mathrm{s})$

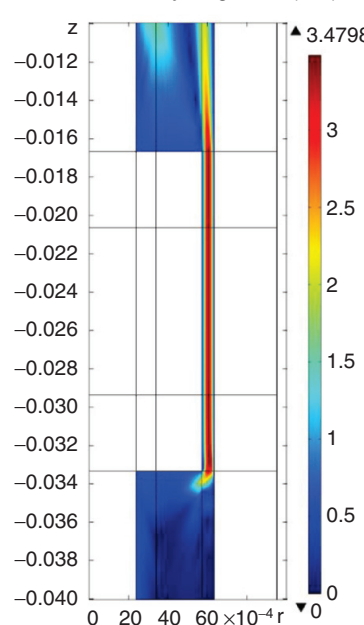

(e)

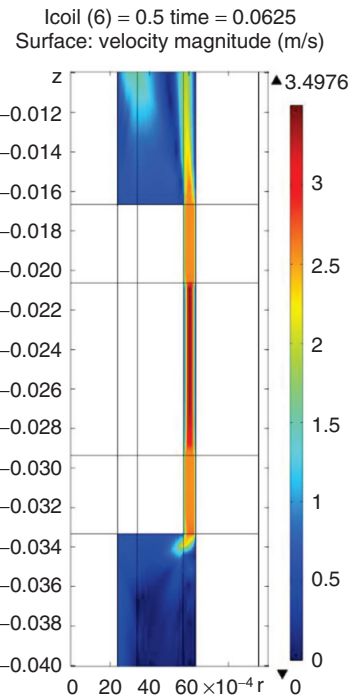

(c)

Icoil $(1)=0$ time $=0.0625$

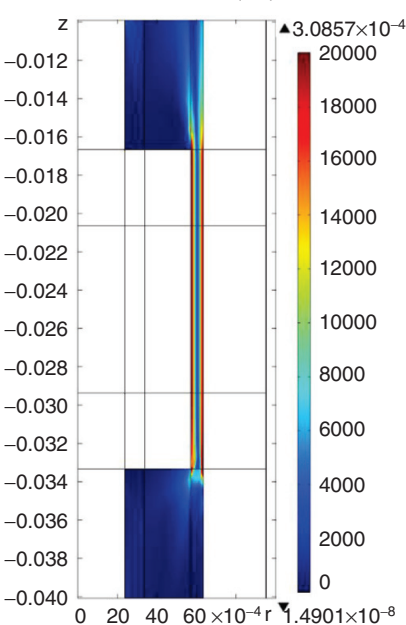

(f)

Icoil $(6)=0.5$ time $=0.0625$ Surface: shear rate $(1 / \mathrm{s})$

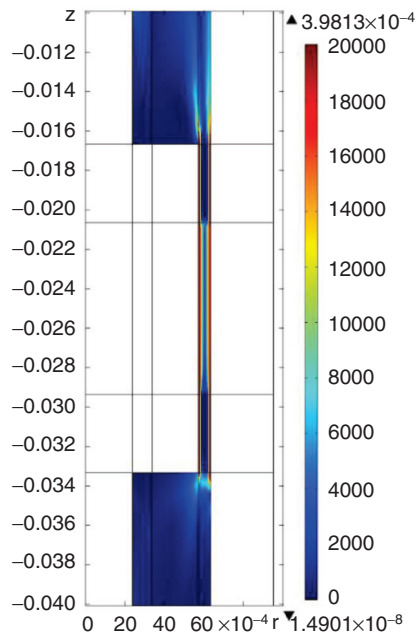

Figure 6 Comparison of model results with $4 \mathrm{~Hz}$ piston motion at peak piston velocity. Magnetic field $(a, d)$, velocity magnitude $(b, e)$, and fluid shear rate $(c, f)$ with $0 \mathrm{~mA}$ (upper) and $500 \mathrm{~mA}$ (lower) applied current. 


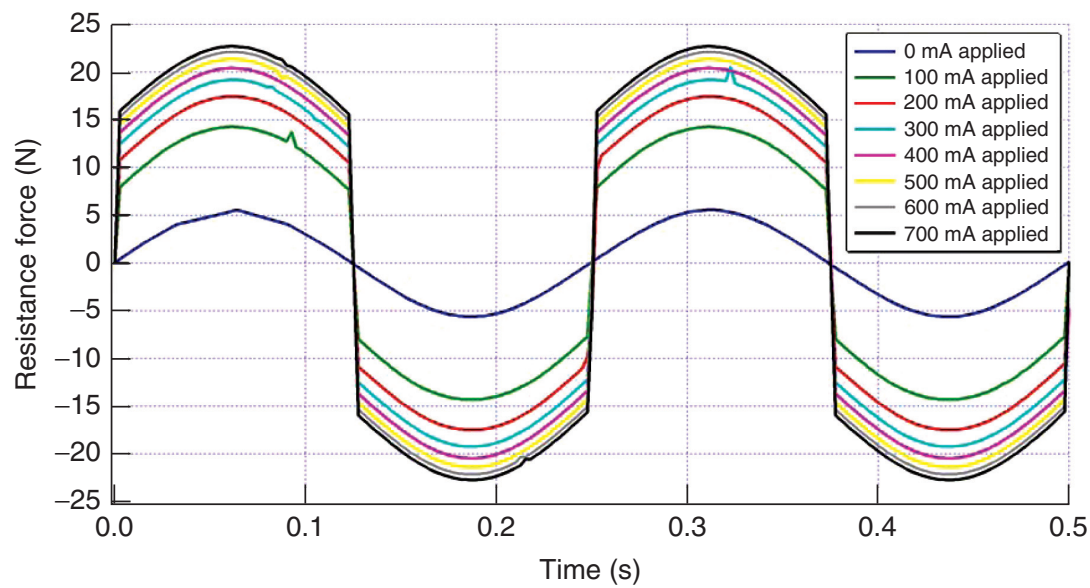

Figure 7 Resistance forces derived from the dynamic FEM model of the damper. Piston motion is cyclic at $4 \mathrm{~Hz}$ and $25 \mathrm{~mm}$ amplitude with applied current in the range of 0-700 mA.

Stress in the axial direction was integrated over the surfaces of the rod and piston to calculate the total resistance force of the damper. Results are shown in Figure 7 for the $4 \mathrm{~Hz}$ sinusoidal motion.

\section{EXPERIMENTAL VALIDATION}

Experimental validation of the force response of the damper under cyclic motion was performed on a custom experimental setup, designed to convert the rotation of a brushless DC motor (EDC, Cambridge, MA) to sinusoidal translation of the piston of the damper via a crank mechanism and a linear bearing (Figure 8). A force transducer (MLP-50, Transducer Techniques, Temecula, CA) and a position sensor (MLT-38000104, Honeywell, Morristown, $\mathrm{NJ}$ ) were used to measure dynamic behavior of the damper, and pressure sensors (ASCX100AN, Honeywell, Morristown, NJ) were used to monitor the internal fluid pressure differential. An optical encoder (ACCU-Coder 260 N-T-02-S-1000, Encoder Products Co.,

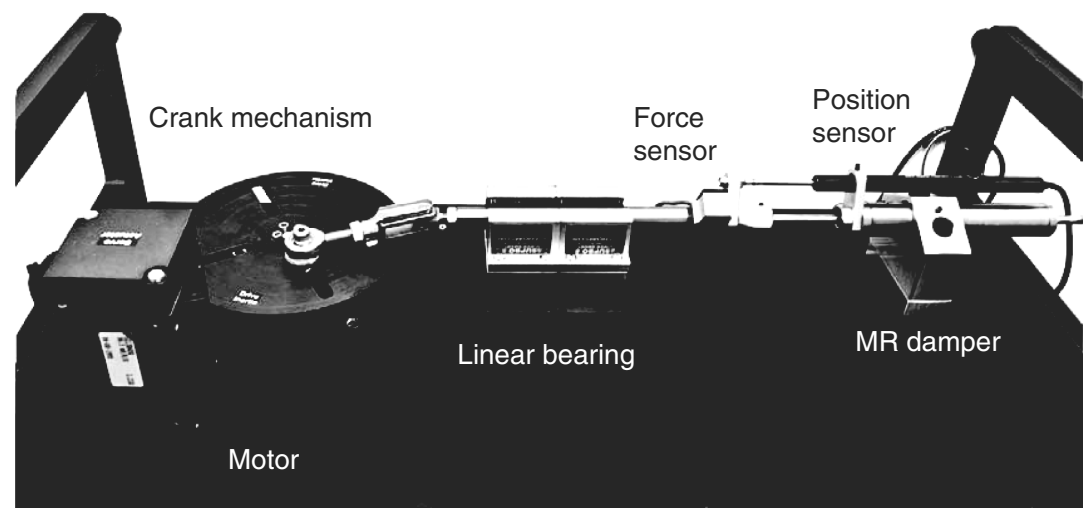

Figure 8 Experimental setup for FE analysis validation of dynamic behavior of the MR damper. 
Sandpoint, ID) was used to measure the angular position of the crank mechanism. A multifunction RIO NI PCI-7833R FPGA card was employed for real-time data acquisition and control of the electrical motor. In order to mitigate the discrete nature of the optical encoder readout, the angular velocity and acceleration were estimated in real time using a custom-developed algorithm implemented in the internal memory of the FPGA card.

A set of experiments was performed with the same sinusoidal motions employed in the FEM simulations applied to the piston of the MR damper. The coil current was set to constant values in the range of $0-636 \mathrm{~mA}$, similar to the values used in the simulations. The same set of cyclic motions and coil currents was applied to the damper with no fluid in the internal chamber. The resistance force measured from these experiments includes the force due to friction and piston inertia. A sample of the experimental data is presented in Figure 9.

(a)

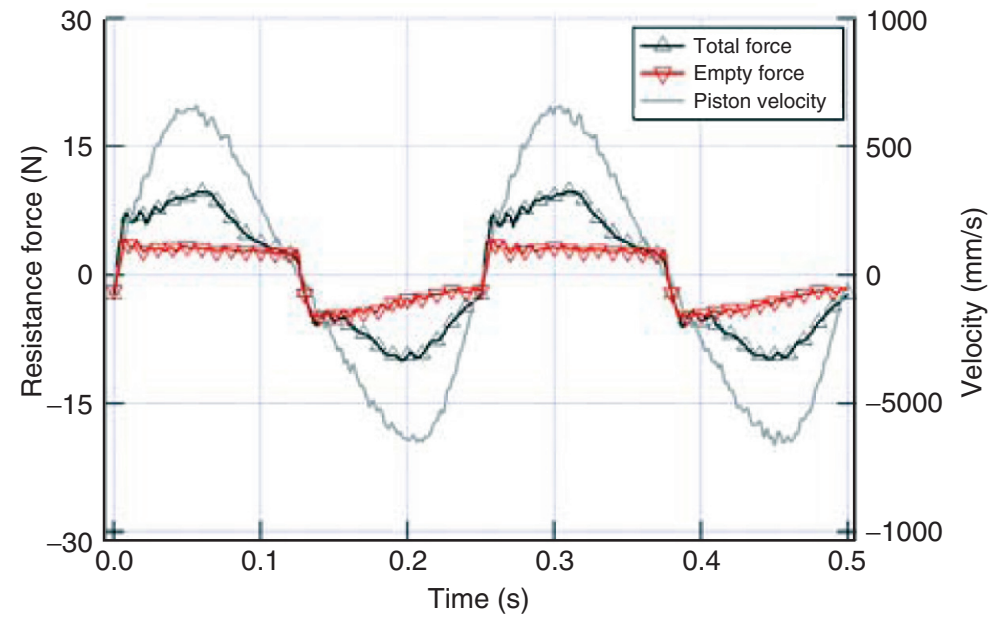

(b)

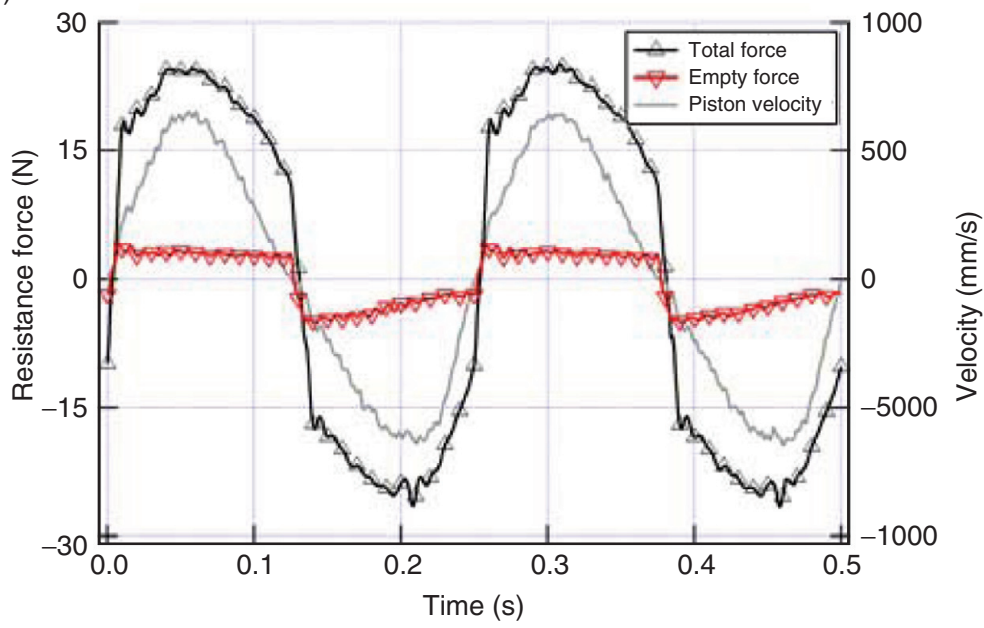

Figure 9 Sample of the data collected with the experimental setup. Cyclic motion at $4 \mathrm{~Hz}$ and $50 \mathrm{~mm}$ stroke length with $0 \mathrm{~mA}(\mathrm{a})$ and $500 \mathrm{~mA}$ (b) applied current. 
In order to estimate the force due strictly to the MR fluid dynamics, resistance force measured from the empty damper was subtracted from the total resistance force with MR fluid. A comparison of the experimental MR fluid force, thus derived, with results from the associated FEM simulation is shown in Figure 10 for two applied coil currents and $4 \mathrm{~Hz}$ cyclic motion. Very good agreement is observed between the FEM and experimental data with and without applied current. The slight profile difference for force measurements can be attributed to the imperfect sinusoidal motion applied to the damper in the experimental data as seen in the velocity curves in Figure 10.

To assess overall fidelity of the FEM model, the experimental and simulated peak MR fluid resistance forces under a broad range of cyclic motions $(0.5-6 \mathrm{~Hz})$ and coil currents $(0-700 \mathrm{~mA})$ were compared (see Figure 11). A strong correlation can be observed between

(a)

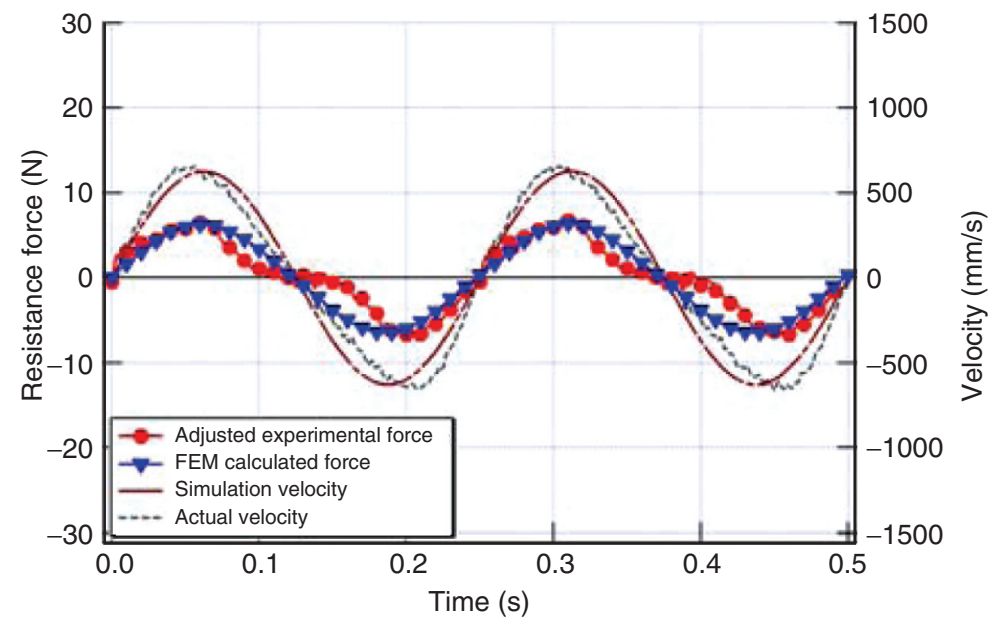

(b)

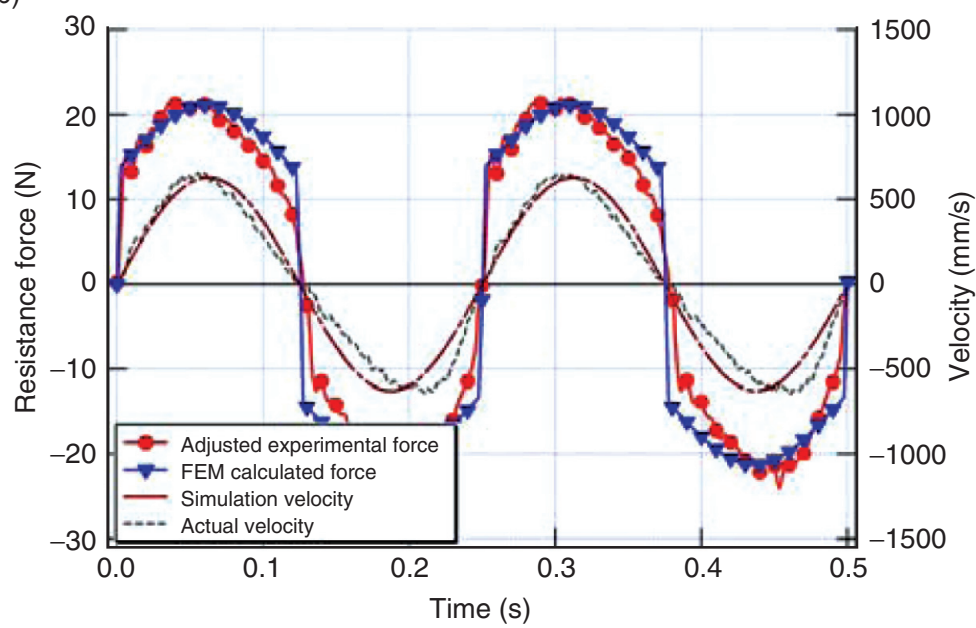

Figure 10 Comparison of experimental and FEM-based resistance force and piston velocity. Cyclic motion at $4 \mathrm{~Hz}$ and $50 \mathrm{~mm}$ stroke length with $\mathrm{O} \mathrm{mA}$ (a) and 500 mA (b) applied current. 
(a)

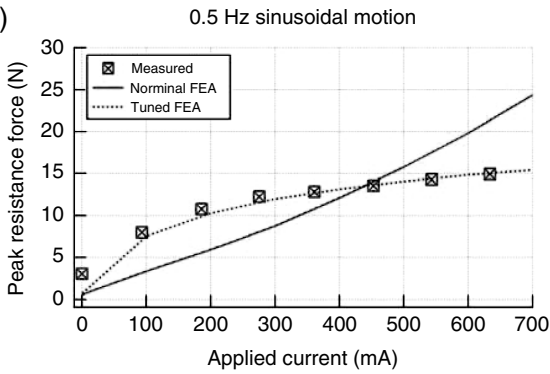

(c)

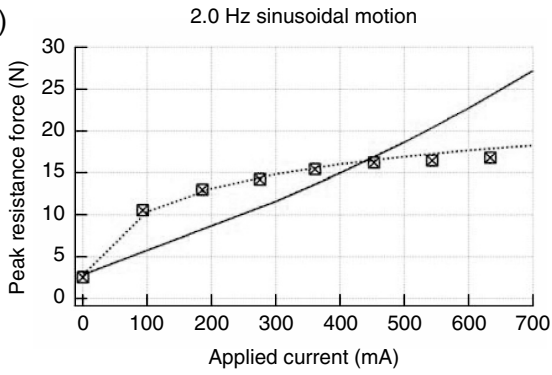

(e)

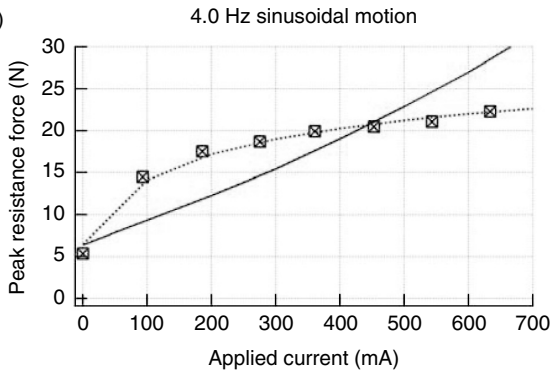

(b)

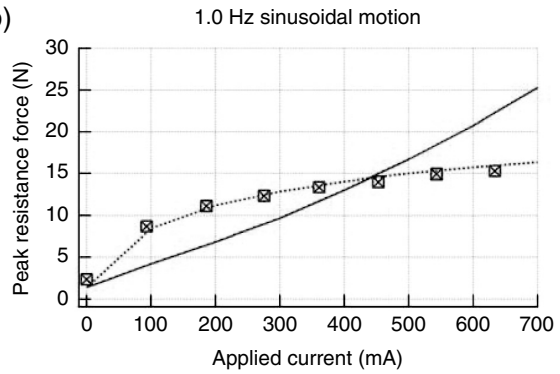

(d)
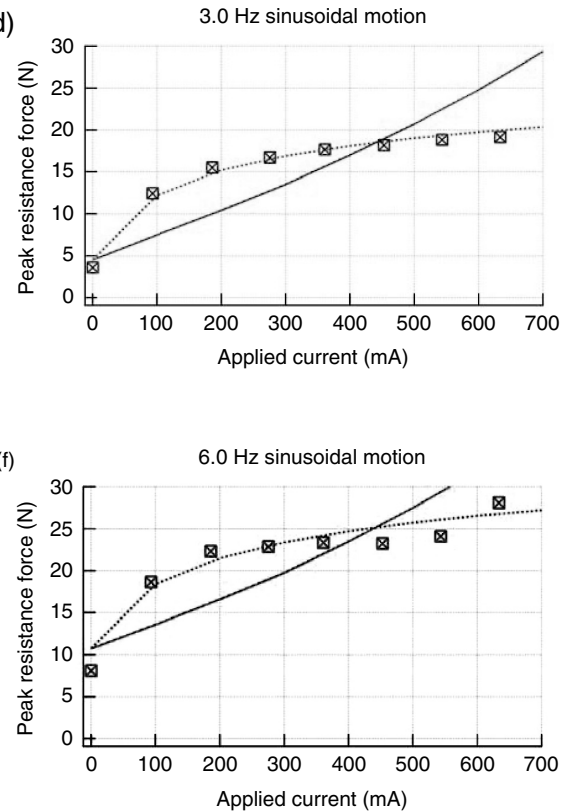

Figure 11 Peak resistance force vs. applied current in experiments and FEA simulations with nominal and experimentally tuned magnetic properties of the fluid. Values are reported for a stroke length of $50 \mathrm{~mm}$ at frequencies of $0.5 \mathrm{~Hz}(\mathrm{a})$, $1.0 \mathrm{~Hz}(\mathrm{~b}), 2.0 \mathrm{~Hz}(\mathrm{c}), 3.0 \mathrm{~Hz}(\mathrm{~d}), 4.0 \mathrm{~Hz}(\mathrm{e})$, and $6.0 \mathrm{~Hz}$ (f).

the experimental data and the values obtain from FEA simulations using the experimentallyderived relationship between fluid yield strength and magnetic field intensity presented previously. In contrast, the results based on the manufacturer's nominal fluid properties exhibit a relatively large error compared to the experimental results.

\section{DISCUSSION AND CONCLUSIONS}

A custom-designed MR damper was analyzed theoretically and experimentally for suitability of use in a low-profile tremor suppression orthosis. A modified stress tensor definition, more adequate for numerical simulations, was introduced for the purpose of modeling of a Bingham plastic-like fluid under non-steady flow conditions. This definition was applied to a time-dependent FEM model, designed to simulate and predict the behavior of the damper under cyclic motion. 
The nominal $\sigma_{y} H$ curve from the MR fluid specifications produced simulation results that showed large errors in comparison to the experimental results. A modified $\sigma_{y} H$ curve, derived from static analysis, produced FEA simulation results that exhibited strong correlation with experimental data under all examined cycling conditions and applied coil currents of the damper.

The multiphysics FEA modeling process described in this paper was deemed suitable for the prediction of oscillatory MR fluid behavior and thus for further development and optimization of the semi-active dampers required for a low-profile tremor suppression orthosis.

\section{REFERENCES}

[1] D. J. Klingenberg, "Magnetorheology: Applications and challenges," AIChE Journal, vol. 47, no. 2, pp. 246-249, February 2001.

[2] G. Yang, B. F. Spencer Jr., J. D. Carlson, and M. K. Sain, "Large-scale mr fluid dampers: modeling and dynamic performance considerations." Engineering Structures, vol. 24, p. 309, 2002.

[3] J. Rosen, S. Burns, and J. Perry, "Upper-limb powered exoskeleton design," IEEE/ASME Transactions on Mechatronics, vol. 12, no. 4, pp. 408-417, 2007.

[4] H. Gurocak and J. Blake, "Haptic glove with MR brakes for virtual reality," IEEE/ASME Transactions on Mechatronics, vol. 14, no. 5, pp. 606-615, 2009.

[5] A. Shafer and M. Kermani, "On the feasibility and suitability of MR fluid clutches in humanfriendly manipulators," IEEE/ASME Transactions on Mechatronics, vol. 16, no. 6, pp. 1073-1082, December 2011.

[6] D. Case, B. Taheri, and E. Richer, "Design and characterization of a small-scale magnetorheological damper for tremor suppression," IEEE/ASME Transactions on Mechatronics, vol. 18, no. 1, pp. 96-103, February 2013, doi: 10.1109/TMECH.2011.2151204.

[7] R. Ehrgott and S. Masri, "Modelling the oscillatory dynamic behavior of electrorheological materials in shear," Smart Materials and Structures, vol. 1, no. 4, pp. 275-285, 1992.

[8] B. Spencer Jr., S. Dyke, M. Sain, and J. Carlson, "Phenomenological model of a magnetorheological damper," ASCE Journal of Engineering Mechanics, vol. 123, no. 3, pp. 230-238, 1997.

[9] T. C. Papanastasiou, "Flow of materials with yield," Journal of Rheology, vol. 31, pp. 385-404, 1987.

[10] A. Taylor and S. Wilson, "Conduit flow of an incompressible yield stress fluid," Journal of Rheology, vol. 41, no. 1, pp. 93-101, 1997.

[11] G. Burgos, A. Alexandrou, and V. Entov, "On the determination of yield surfaces in herschelbulkley fluids," Journal of Rheology, vol. 43, no. 3, pp. 463-483, 1999.

[12] I. Frigaard and C. Nouar, "On the usage of viscosity regularization methods for visco-plastic fluid flow computation," Journal of Non-Newtonian Fluid Mechanics, vol. 127, pp. 1-26, 2005.

[13] M. Moyers-Gonzalez and I. Frigaard, "Numerical solution of duct flows of multiple visco-plastic fluids," Journal of Non-Newtonian Fluid Mechanics, vol. 122, pp. 227-241, 2004.

[14] R. Phillips, Engineering applications of fluids with a variable yield stress, Ph.D. dissertation, Berkeley, 1969.

[15] G. M. Kamath, M. K. Hurt, and N. M. Wereley, "Analysis and testing of bingham plastic behavior in semi-active electrorheological fluid dampers," Smart Materials and Structures, vol. 5, pp. 576-590, 1996. 
[16] J. G. Oldroyd, "A rational formulation of the equation of plastic flow for a Bingham solid," Proceedings of the Cambridge Philosophical Society, vol. 43, pp. 100-105, 1947.

[17] A. Cantelli, "Uniform flow of modified Bingham fluids in narrow cross sections," Journal of Hydraulic Engineering, vol. 135, no. 8, pp. 640-650, 2009 stress," Ph.D. dissertation, Berkeley, 1969. 